\title{
3D finite element analysis of Al7075-T6 drilling with coated solid tooling
}

\author{
Anastasios Tzotzis ${ }^{1}$, Angelos Markopoulos ${ }^{2}$, Nikolaos Karkalos ${ }^{2}$ and Panagiotis Kyratsis ${ }^{3,}$ \\ ${ }^{1}$ University of Zaragoza, Department of Design and Manufacturing, C/Maria de Luna 3, 50018 \\ Zaragoza, Spain \\ ${ }^{2}$ National Technical University of Athens, School of Mechanical Engineering, Heroon Polytechniou \\ 9, 15780 Zografou, Athens, Greece \\ ${ }^{3}$ University of Western Macedonia, Dept. of Product and Systems Design Engineering, Kila Kozani, \\ GR50100, Greece
}

\begin{abstract}
Due to the fact that simulation of drilling was added in commercial finite element analysis (FEA) software only recently, 3D finite element modelling is an invaluable asset during related researches. The present study employs 3D FEA to model the drilling process of Al7075-T6 alloy with solid carbide tooling, investigates important phenomena that occur during drilling and finally compares the simulated results with experimental data. A number of simulations were performed with DEFORM3D ${ }^{\mathrm{TM}}$ software at different cutting conditions; cutting speed of $50 \mathrm{~m} / \mathrm{min}, 100 \mathrm{~m} / \mathrm{min}, 150 \mathrm{~m} / \mathrm{min}$ and feed of $0.15 \mathrm{~mm} / \mathrm{rev}, 0.20 \mathrm{~mm} / \mathrm{rev}$, $0.25 \mathrm{~mm} / \mathrm{rev}$. The proposed model takes into consideration certain aspects like damage initiation and evolution of the material, contact interface between the drill bit and the workpiece and standard boundary conditions. Eventually, the acquired numerical data for thrust force were compared to the experimental results for the same cutting conditions and parameters. To obtain the experimental data, a series of nine drilling tests were performed. Upon validation of the numerical data, the temperature distribution on the tool tip - workpiece interface, as well as the chip morphology (shape and curling radius) were determined. Results showed a good agreement between the numerical and the experimental data. Specifically, thrust force and chip morphology exhibited an agreement of about $95 \%$ and $90 \%$ respectively, which confirms the potential of 3D FEA implementation on machining investigations.
\end{abstract}

\section{Introduction}

Since drilling is one of the most used machining process, its study draws the attention of many researchers worldwide. Especially, the implementation of modern techniques and tools such as the finite element method (FEM) during the research of drilling is a topic with increasing interest.

Lotfi et al. [1] developed a 3D finite element (FE) model to investigate the heat and wear on the drill faces during drilling process of AISI 1045 steel. Moreover, authors verified the

\footnotetext{
*Corresponding author: pkyratsis@uowm.gr
} 
simulated model with experiments and evaluated the surface roughness and built-up edge. Dou et al. [2] developed a new constitutive model to improve a model for prediction purposes of generated thrust force and torque in drilling. Additionally, a 3D FE model for simulating the drilling process of SiCp/Al6063 was established by implementing the new constitutive model. Finally, authors compared the numerical results with the experimental observations in thrust force, torque, and chip morphology. Similarly, Xiang et al. [3] analyzed in their work the micro mechanisms underlying the wear of macroscale tools during diamond machining of SiCp/Al6063 composites. Moreover, they developed the mechanism-based diamond wear model in relation to the dominant wear behaviors. Majeed et al. [4] investigated the effect of drill bit temperature on tool life of twist drills during drilling of AISI 4340 high-strength steel both numerically and experimentally. The 3D FE modelling of the hard drilling process was performed with DEFORM3D ${ }^{\mathrm{TM}}$ software. Oezkaya et al. [5] presented a 3D FEM simulation model to predict the performance of modified flow drilling tools. In addition, to validate the simulation model, authors investigated the flow drilling of AlSi10Mg with a non-pre-heated and pre-heated tool as well. Zhu et al. [6] conducted a new 3D schematic to fully understand the chip formation and morphology in dry drilling of Ti6A14V alloys in order to supply a better understanding of chip formation theory and metallurgical properties in chips.

Even though many researchers in the past few years utilized 3D FE modelling for investigation purposes of machining, 3D analysis of alloy drilling is a topic with multiple aspects that require thorough examination. In this paper, 3D FEA is implemented in order to study the behavior of A17075-T6 alloy during conventional drilling process with coated solid carbide tools. In particular, the thrust force, the tool tip - workpiece temperature distribution and the chip cone radius were determined.

\section{Methodology}

\subsection{Experimental process setup}

A set of nine drilling experiments were carried out with the utilization of HAAS VF1 CNC machining center. A plate of Al7075-T6 alloy with dimensions $150 \mathrm{~mm} \times 130 \mathrm{~mm} \times 15 \mathrm{~mm}$ was used as the workpiece. A $10 \mathrm{~mm}$ solid carbide drill from Kennametal with catalogue number B041A1000CPG (KC7325 grade), was used to perform the experiments. The drills of this series are double coated; they have a multilayer of TiN/TiAlN with 3.5 micron thickness and an outer layer of TiN with 15 micron thickness. Figure 1 illustrates some of the most important parameters of the tool. Finally, in order to measure the produced thrust forces, a Kistler type 9257B 3-component dynamometer was configured.

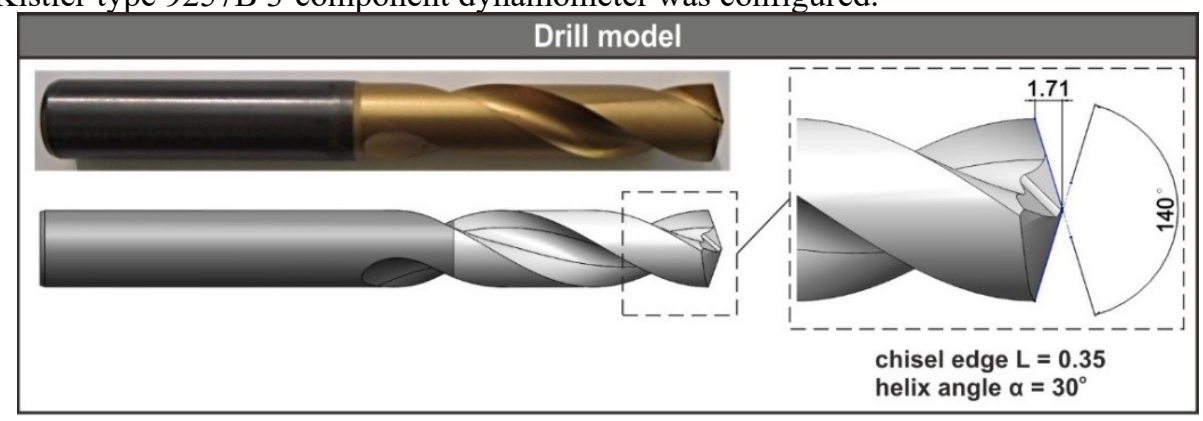

Fig. 1. Basic geometric characteristics of the B041A1000CPG drill. 


\subsection{Simulation setup}

The 3D simulations of the nine drilling experiments were performed with DEFORM3D ${ }^{\mathrm{TM}}$ ver. 11.3 simulation software by maintaining the same execution order as the experiments. Moreover, the simulations were stopped when the thrust force achieved steady state for time saving purposes. The model of the workpiece was designed to be circular and thin, with a thickness equal to the radius of the drill. To further reduce the simulation time, a center drill spot based on the point angle of the tool was created on the workpiece (Figure 2a).

The workpiece was modelled as plastic and meshed with tetrahedral elements. The minimum element size of the mesh was set to $50 \%$ of the feed [7]. Furthermore, the mesh at the center of the workpiece was refined with a ratio of 10:1 (Figure 2a), so that the contact interface between the tool tip and the workpiece surface can be optimized. Due to the fact that the area located at the center of the workpiece is where high temperatures, large strains and deformations occur, an adaptive remeshing method was employed. In this way, the simulation time was improved as well as the formation of the chip.

To simplify the tool modelling, only the tip of the tool was used. It was modelled as rigid and meshed with approximately 25000 tetrahedral elements. A finer mesh with a 4:1 ratio was applied closer to the tip (Figure 2b). Finally, both the model of the workpiece and the drill were converted to STL file type prior to importing to DEFORM3D ${ }^{\mathrm{TM}}$.

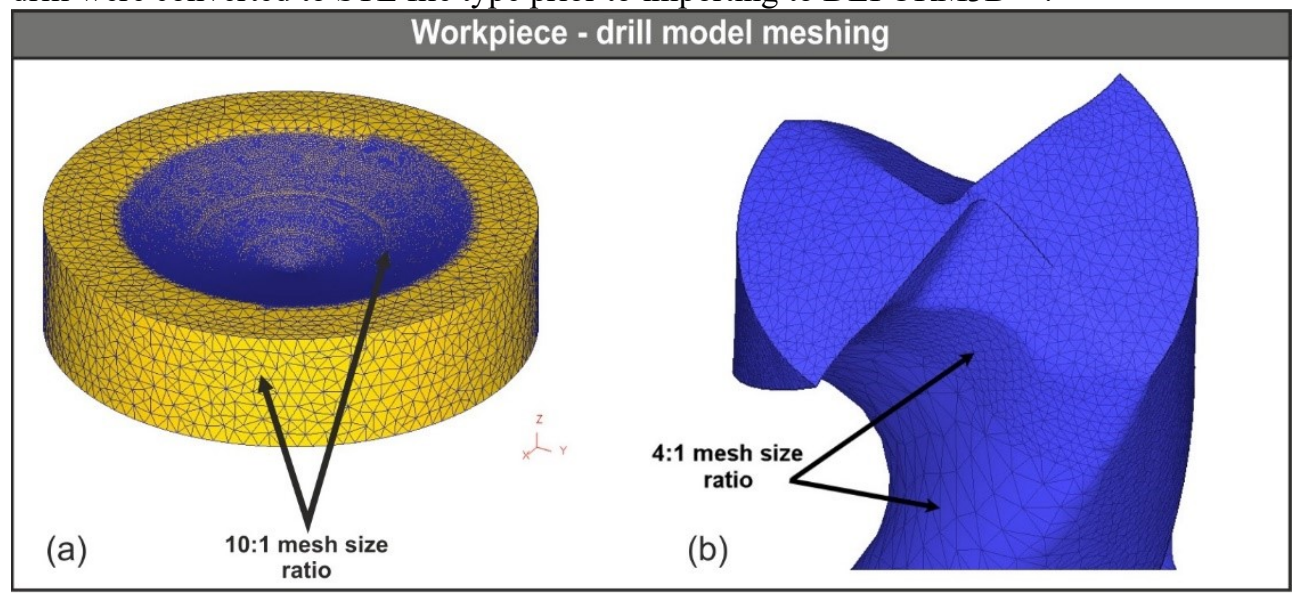

Fig. 2. The workpiece - drill model mesh setup.

In addition to the normal meshing of the tool, an extra layer of mesh was applied, so that the double coating of the drill can be taken into account to the numerical results. The basic properties of Al7075-T6 alloy, TiN and TiAlN coatings are presented in Table 1.

Table 1. Basic properties of workpiece material [8] and tool coatings.

\begin{tabular}{c|ccc}
\hline Material & Al7075-T6 & TiN & TiAlN \\
Young's modulus $(\mathrm{GPa})$ & 71.7 & 60 & 44 \\
Density $\left(\mathrm{kg} / \mathrm{m}^{3}\right)$ & 2810 & 5220 & 5220 \\
Poisson's ratio & 0.33 & 0.25 & 0.23 \\
Thermal expansion & $2.2 \times 10^{-5}$ & $9.4 \times 10^{-6}$ & $9.2 \times 10^{-6}$ \\
$\left(\mu \mathrm{m} / \mathrm{m}^{\circ} \mathrm{C}\right)$ & & & \\
Thermal conductivity & 130 & 25 & 12 \\
$@ 20^{\circ} \mathrm{C}(\mathrm{W} / \mathrm{mK})$ & & & \\
\hline
\end{tabular}


The generalized Johnson - Cook model was selected for the simulation of the flow stress of A17075-T6 during drilling since in such operations deformation is high. This model takes into consideration the effect of three parameters: the effect of strain, strain rate and temperature. The model can be described by equation 1 .

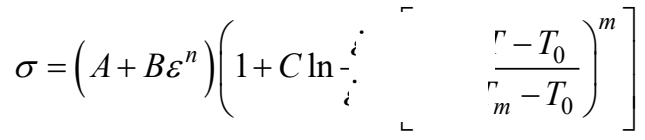

The parameters that form equation 1 are: $A$ (initial yield stress), $B$ (strain hardening modulus), $C$ (strain rate dependence coefficient), $\varepsilon$ (plastic strain), $n$ (strain hardening exponent), $m$ (thermal softening coefficient), $\dot{\varepsilon}$ (plastic strain rate), $\dot{\varepsilon}_{0}$ (reference plastic strain rate), $T$ (reference temperature), $T_{0}$ (bulk temperature of workpiece material) and $T_{m}$ (melting temperature of workpiece material). Table 2 contains the values of the parameters and constants for A17075-T6. In this study, a reference strain rate of 1/s was used.

Table 2. The constitutive model parameters and constants for Al7075-T6 [9].

\begin{tabular}{ccccccc}
\hline $\begin{array}{c}\mathrm{A} \\
(\mathrm{MPa})\end{array}$ & $\begin{array}{c}\mathrm{B} \\
(\mathrm{MPa})\end{array}$ & $\mathrm{C}$ & $\mathrm{n}$ & $\mathrm{m}$ & $\begin{array}{c}\mathrm{T}_{0} \\
\left({ }^{\circ} \mathrm{C}\right)\end{array}$ & $\begin{array}{c}\mathrm{T}_{\mathrm{m}} \\
\left({ }^{\circ} \mathrm{C}\right)\end{array}$ \\
\hline 546 & 678 & 0.024 & 0.71 & 1.56 & 20 & 635 \\
\hline
\end{tabular}

To simulate the phenomenon of material separation, the damage model of Cockcroft and Latham was employed [9]. In addition, to define the tool - chip interface, a hybrid model (Shear - Coulomb) [10] was used for friction. This model approximates the developed frictional stresses around the tool tip and at the same time describes the friction across the sliding zone. For this study, the constant shear friction coefficient is $\mathrm{m}=0.7$ and the constant Coulomb friction coefficient is $\mu=0.6$.

The nodes at the side of the workpiece were fixed in all three axes $(X, Y, Z)$ so that velocity is zero and thus the workpiece is treated as stationary. On the other hand, a constant rotation around $\mathrm{Z}$ axis and a constant feed towards $-\mathrm{Z}$ axis were assigned to define the rotational and translational movement of the tool. The boundary conditions that relate to the heat exchange with the environment were defined and in addition, heat transfer through convection between the workpiece and the tool tip surface was defined. The default convection coefficient for dry cutting was used with a value of $0.02 \mathrm{~N} / \mathrm{sec} / \mathrm{mm} /{ }^{\circ} \mathrm{C}$. Moreover, to approximate the heat transfer through conduction in the tool - workpiece interface, the default coefficient was used with a value of $45 \mathrm{~N} / \mathrm{sec} / \mathrm{mm} /{ }^{\circ} \mathrm{C}$.

\section{Experimental and simulated results}

\subsection{Assessment of thrust force}

The nine simulations were performed on a desktop PC with dual-core CPU, 16GB RAM and SSD hard drive. The produced values of thrust force for each set were exported to Excel ${ }^{\mathrm{TM}}$ for processing and comparison with the experimental results. Figure 3 depicts a sample thrust force diagram (lower left diagram) at cutting conditions of $V_{c}=50 \mathrm{~m} / \mathrm{min}$ and $f=0.15 \mathrm{~mm} / \mathrm{rev}$. It is concluded that thrust force increases rapidly and reaches a steady state quickly. In this case, the time before thrust force stops to increase and steady state begins is approximately $0.0171 \mathrm{sec}$. Similar trend was observed in all simulations. The values of thrust force varied between 370 and $605 \mathrm{~N}$ for the aforementioned cutting conditions, whereas the mean value was calculated roughly $500 \mathrm{~N}$. 


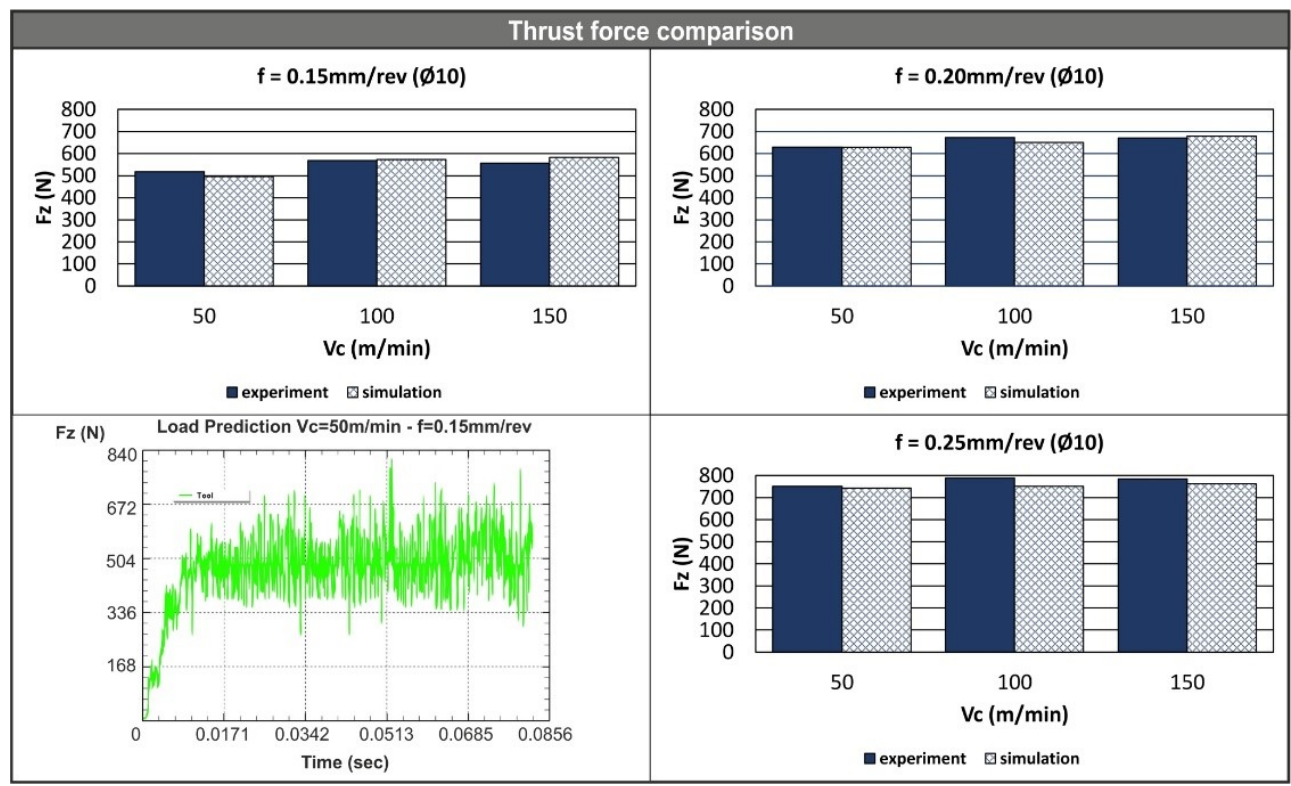

Fig. 3. Comparison between the experimental and the simulated thrust forces.

With the process of the simulated thrust force results and the acquisition of the experimental ones, a comparison between them was made. In addition, to the sample thrust force diagram, Figure 3 includes three bar charts that display this comparison for each of the three feed values.

In general, the results of thrust force are in high accordance with the experimental values. The agreement percentages vary between 96 and $102 \%$, whereas in the cases with $V_{c}=50 \mathrm{~m} / \mathrm{min}$ and $f=$ 0.20 and $0.25 \mathrm{~mm} / \mathrm{rev}$ the percentage reaches $99 \%$. The maximum deviation between the experimental and the numerical results was found to be in the simulation with $V_{c}=100 \mathrm{~m} / \mathrm{min}$ and $f=0.25 \mathrm{~mm} / \mathrm{rev}$. The default first order exponential smoothing was applied to the results and the standard deviation was calculated between 20 and $75 \mathrm{~N}$. Specifically, the minimum value of standard deviation was found on the simulation with the next conditions: $V_{c}=50 \mathrm{~m} / \mathrm{min}$ and $f=0.15 \mathrm{~m} / \mathrm{min}$, whereas the simulation test with cutting speed of $V_{c}=50 \mathrm{~m} / \mathrm{min}$ and feed of $f=0.25 \mathrm{~mm} / \mathrm{rev}$ exhibited the maximum value of standard deviation. Furthermore, the mean value of standard deviation for all nine simulation tests was determined $52 \mathrm{~N}$.

\subsection{Assessment of chip formation and temperature distribution}

The formation of chip in drilling of A17075-T6 follow a similar pattern for both the experimental and the simulated procedure. Figure $4 \mathrm{a}$ depicts the experimentally generated chip at cutting speed of $V_{c}=$ $50 \mathrm{~m} / \mathrm{min}$ and feed of $f=0.15 \mathrm{~mm} / \mathrm{rev}$. It is noticeable that the chip has a conical shape which was maintained on all experiments despite the change in feed and cutting speed. Additionally, the same chip morphology was observed for all simulation tests (Figure 4b). Moreover, the diameter of the chip's curling was found to be in good agreement between the experimental and the simulated one. As depicted in Figure $4 \mathrm{~b}$, it was determined approximately $5.33 \mathrm{~mm}$ for the simulation and $6.05 \mathrm{~mm}$ for the experiment, for the same cutting conditions and after approximately 4500 steps (this is when a full curling of the chip formed). The agreement percentage for this case is $86.5 \%$.

Lastly, temperature distribution on the tool tip surface was introduced, due to the heat transfer between the tool and the workpiece. Figure $4 \mathrm{c}$ illustrates the temperature distribution on the $10 \mathrm{~mm}$ tool tip (B041A1000CPG) after about 8182 steps $(\approx 0.1 \mathrm{~mm}$ penetration depth). It is obvious that the maximum temperature of around $67.9^{\circ} \mathrm{C}$ is located on the cutting lips. A slight increase in maximum temperature was observed as higher values of cutting speed and especially feed were chosen. The simulations were intentionally stopped after a number of steps in order to avoid very long completion times. Before a simulation was halted, it was allowed to reach steady state and ensured that it was maintained for a reasonable period. 


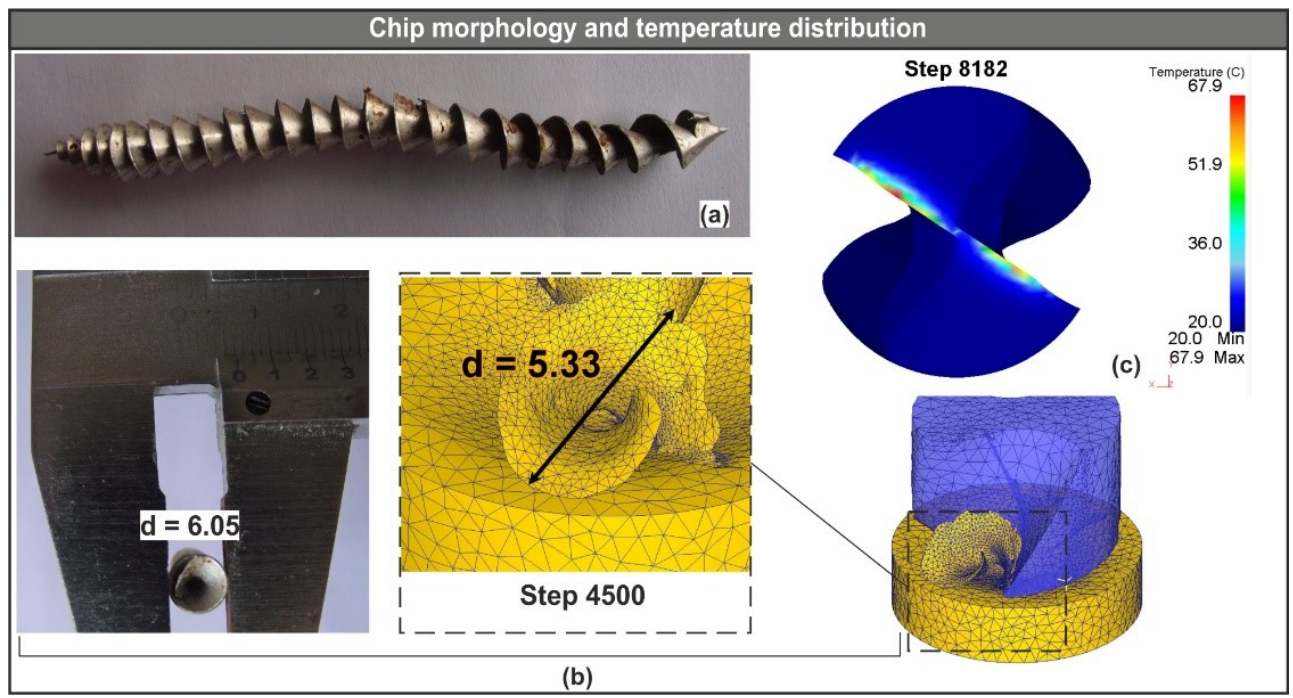

Fig. 4. Chip morphology comparison and temperature distribution on the tool tip.

\section{Conclusions}

Nine 3D simulations of Al7075-T6 drilling process with a $10 \mathrm{~mm}$ solid carbide tool were performed under different cutting conditions; cutting speed between 50 and $150 \mathrm{~m} / \mathrm{min}$ and feed between 0.15 and $0.25 \mathrm{~mm} / \mathrm{rev}$. The simulated results were validated with the implementation of experimental testing and the findings demonstrated an increased correlation between the simulated and the experimental results (between 96\% and 102\%). Moreover, the morphology and the dimensions of the chip during formation further confirm the validity of the 3D FE model presented in this paper. Subsequently, the findings of this study can lead to the following conclusions:

- An increase in cutting speed can increase thrust force by a small value.

- On the other hand, an increase in feed have significant impact in the produced thrust force.

- The generated chip morphology retains a typical conical shape which do not alter with a change on the cutting conditions.

- The maximum developed temperature on the drill is located on the cutting lips.

\section{References}

1. M. Lotfi, S. Amini, and I. Y. Al-Awady, Adv. Manuf., 6, 2, pp. 204-214, (2018)

2. T. Dou, H. Fu, Z. Li, X. Ji, and S. S. Bi, Int. J. Adv. Manuf. Technol., 103, 1-4, (2019)

3. J. Xiang, S. Pang, L. Xie, F. Gao, X. Hu, J. Yi and F. Hu, Materials (Basel), 11, 2, (2018)

4. A. Majeed, A. Iqbal, and J. Lv, Int. J. Adv. Manuf. Technol., 95, 5-8, (2018)

5. E. Oezkaya, S. Hannich, and D. Biermann, Int. J. Mater. Form., 12, 3, (2019)

6. Z. Zhu, K. Gao, J. Sun, J. Li, Y. Liu, L. Chen and Y. Zheng, J. Manuf. Process., 34, pp. 531-539, (2018)

7. SFTC, "DEFORM V11.3 (PC) Documentation." (2016)

8. P. Kyratsis, A. Markopoulos, N. Efkolidis, V. Maliagkas, and K. Kakoulis, Machines, 6, 2, (2018)

9. M. G. Cockcroft and D. J. Latham, J. Institue Met., 96, pp. 33-39, (1968)

10. İ. Ucun, J. Mech. Sci. Technol., 30, 4, (2016) 